\title{
Principales Criterios de Diagnóstico de la Nueva Clasi- ficación de Enfermedades y Condiciones Periodontales
}

\author{
Main Diagnostic Criteria of the New Classification \\ of Conditions and Periodontal Diseases
}

\begin{abstract}
Paola Cárdenas Valenzuela1; Dalia Abril Guzmán Gastelum²; Eligio Valera González²; Juan Carlos Cuevas González²; Graciela Zambrano Galván³ \& Alma Graciela García Calderón²
\end{abstract}

CÁRDENAS, V. P.; GUZMÁN, G. D. A.; VALERA, G. E. ; CUEVAS, G. J. C.; ZAMBRANO, G. G. \& GARCíA, C. A. G. Principales criterios de diagnóstico de la nueva clasificación de enfermedades y condiciones periodontales. Int. J. Odontostomat., 15(1):175-180, 2021.

RESUMEN: Tener una clasificación de enfermedades periodontales es de gran utilidad para los clínicos al brindar una guía sistematizada de las distintas manifestaciones de los problemas periodontales. La nueva clasificación de enfermedades y condiciones periodontales de 2017 tiene como una de sus características principales la agrupación de la periodontitis con estadios y grados, como manifestación de enfermedades sistémicas y enfermedades periodontales necrosantes; así como la introducción de un apartado que habla de salud y enfermedad peri-implantaria. En el caso de la periodontitis, el estadio se refiere a la severidad de la enfermedad y la complejidad del tratamiento; mientras que el grado se refiere al ritmo del progreso y riesgo de recidiva de la enfermedad, en este trabajo se ofrece una ruta de diagnóstico didáctica y se desarrollan las principales diferencias entre las últimas dos clasificaciones, al tiempo que compartimos la experiencia al implementar esta nueva clasificación en nuestro instituto.

PALABRAS CLAVE: periodontitis periapical, periodontitis, periodontitis crónica.

\section{INTRODUCCIÓN}

Los sistemas de clasificación de enfermedades periodontales agrupan condiciones que van desde la gingivitis hasta los distintos estados de la periodontitis y actualmente, las condiciones peri-implantares. Estos han sido modificados y actualizados para que los clínicos puedan tener diagnósticos adecuados y el tratamiento sea óptimo (Graetz et al., 2019). Desde la primera descripción de la enfermedad periodontal, se han manejado diferentes sistemas de clasificación para agruparlas por su etiología, patogenia, localización y progreso, pero siempre existe alguna particularidad o complicación para realizar el diagnóstico apropiado y personalizado de los pacientes (Dietrich et al., 2019). El objetivo de este trabajo está enfocado en brindar una ubicación sistematizada del diagnóstico individual de cada paciente, siendo esto necesario al existir complicaciones para seguir una ruta correcta, teniendo como base la nueva clasificación de enfermedades y condiciones periodontales del año 2017.

Según la Organización Mundial de la Salud, la enfermedad periodontal afecta a los tejidos que rodean y sostienen al diente; la enfermedad se manifiesta con sangrado e inflamación gingival, siendo la gingivitis su estado inicial. En las fases más avanzadas el tejido gingival pierde su inserción de los dientes y el hueso, lo cual provoca movilidad y pérdida dental; lo que conocemos como periodontitis. La gingivitis tiene dos principales etiologías; inducida o no por placa, esta última se refiere a que puede iniciar debido a en-

\footnotetext{
${ }^{1}$ Estudiante del segundo semestre de la especialidad de periodoncia, Departamento de Estomatología, Instituto de Ciencias Biomédicas, Universidad Autónoma de Ciudad Juárez, Chihuahua, México.

2 Profesor-investigador, Departamento de Estomatología, Instituto de Ciencias Biomédicas, Universidad Autónoma de Ciudad Juárez, Chihuahua, México.

${ }^{3}$ Profesora investigadora, Facultad de Odontología, Universidad Juárez del Estado de Durango, Durango, México.
}

Received: 2020-06-16 Accepted: 2020-08-29 
fermedades sistémicas, malnutrición, infecciones, desórdenes endócrinos, etc. el paciente regresa al estado de salud si se trata adecuadamente y en caso contrario, puede evolucionar a periodontitis (Graetz et al.).

Clasificaciones de 1999 y 2017. Las últimas dos clasificaciones de las enfermedades y condiciones periodontales han sido las de 1999 y 2017 (Tabla I), se han hecho grandes cambios y se han agregado categorías tanto para condiciones gingivales, periodontales y en la última, peri-implantarias. En la de 1999 se organizaron las enfermedades gingivales en 2 grupos: enfermedad inducida y no relacionada a placa, en donde se describieron aquellas condiciones que eran alteradas por factores sistémicos, como perturbaciones en el sistema endócrino, medicamentos y malnutrición entre otros. En el 2017, se conservó esta situación y además se añadieron las condiciones de salud e inflamación en un periodonto reducido después del tratamiento periodontal exitoso; se acordó también que el sangrado al sondeo es el principal parámetro para clasificar los distintos umbrales de la gingivitis, y que esta fase es aún reversible (Armitage, 1999; Caton et al., 2018). La periodontitis que en 1999 se dividió en crónica, agresiva y como manifestación de enfermedades sistémicas, en el 2017 se recategorizó eliminando la crónica y la agresiva, y se designó un solo grupo de periodontitis, pero utilizando estadios y grados. Los estadios se refieren a la severidad de la enfermedad y complejidad del tratamiento
(Estadios I, II, III y IV); mientras que el grado se refiere de las características biológicas como el progreso y severidad (Grados A, B y C) (Dietrich et al.).

En la clasificación de 1999 las enfermedades periodontales necrosantes incluían la gingivitis, periodontitis y estomatitis, como parte de los estadios de la enfermedad, lo cual para el 2017 se decidió mantener sin modificación (Papapanou et al., 2018), lo que si cambió, fue la sustitución del término espesor biológico por el de tejido de inserción supracrestal y la adición de la nueva categoría de condiciones y enfermedades peri-implantares, donde se describe la salud peri-implantar, mucositis peri-implantaria, periimplantitis y deficiencias de tejido blando y óseo en el sitio del implante (Armitage; Caton et al.), otro cambio importante fue con respecto al periodonto reducido, en la clasificación previa se consideraba enfermedad a pesar de que el tratamiento periodontal hubiera sido exitoso, la clasificación de abscesos periodontales y lesiones endo-periodontales se mantuvieron sin cambios ya que ambas tienen como característica la presencia previa de periodontitis (Herrera et al., 2018) (Fig. 1).

Si bien la última clasificación intenta agrupar las enfermedades gingivales y periodontales de una manera más sencilla, es claro que para el dominio de la misma se requiere profundizar en su conocimiento; en nuestro caso en particular en un inicio tanto los docentes y alumnos de pregrado y posgrado presen-

Tabla I. Principales diferencias entre ambas clasificaciones (Adaptado de Graetz et al., 2019; Herrera et al., 2018 y Caton et al., 2018).

\begin{tabular}{|c|c|}
\hline $\begin{array}{c}\text { Gingivitis } \\
1999\end{array}$ & 2017 \\
\hline $\begin{array}{l}\text { G. Asociada a Placa Dentobacteriana } \\
\text { G. Modificada por Factores Sistémicos }\end{array}$ & $\begin{array}{l}\text { G. Asociada al Biofilm } \\
\text { G. No asociada al Biofilm (se incluyeron las últimas } 3 \\
\text { clasificaciones del 1999) }\end{array}$ \\
\hline $\begin{array}{l}\text { G. Modificada por Fármacos } \\
\text { G. Modificada por Malnutrición }\end{array}$ & Agrandamientos Gingivales \\
\hline \multicolumn{2}{|l|}{ Periodontitis } \\
\hline 1999 & 2017 \\
\hline P. Crónica & Periodontitis \\
\hline P. Agresiva & P. Como Manifestación de Enfermedades Sistémicas \\
\hline $\begin{array}{l}\text { P. Como Manifestación de Enfermedades Sistémicas } \\
\text { Enfermedades Periodontales Necrosantes }\end{array}$ & Enfermedades Periodontales Necrosantes \\
\hline $\begin{array}{l}\text { Abscesos Periodontales } \\
\text { P. Asociada a Lesiones Endo-Periodontales }\end{array}$ & *Se agruparon bajo Otras Condiciones Periodontales \\
\hline $\begin{array}{c}\text { Manifestaciones Periodontales de Desarrollo o Adquiridas } \\
1999\end{array}$ & $\begin{array}{l}\text { Enfermedades Sistémicas y Condiciones de } \\
\qquad 2017\end{array}$ \\
\hline Factores Dentales Localizados Retentivos de Placa & $\begin{array}{l}\text { Enfermedades Sistémicas o Condiciones que Afectan al } \\
\text { Periodonto }\end{array}$ \\
\hline Deformidades Mucogingivales Alrededor de los Dientes & $\begin{array}{l}\text { Otras Condiciones Periodontales (Abscesos Periodontales } \\
\text { y Lesiones Endo-Periodontales) }\end{array}$ \\
\hline Deformidades Mucogingivales en Zonas Edéntulas & Deformidades Mucogingivales Alrededor de los dientes \\
\hline Trauma Oclusal & $\begin{array}{l}\text { Fuerzas Oclusales Traumáticas (Incluyen Ortodoncia) } \\
\text { Factores Dentales y P rotésicos que Predisponen a las } \\
\text { Enfermedades Gingivales }\end{array}$ \\
\hline
\end{tabular}



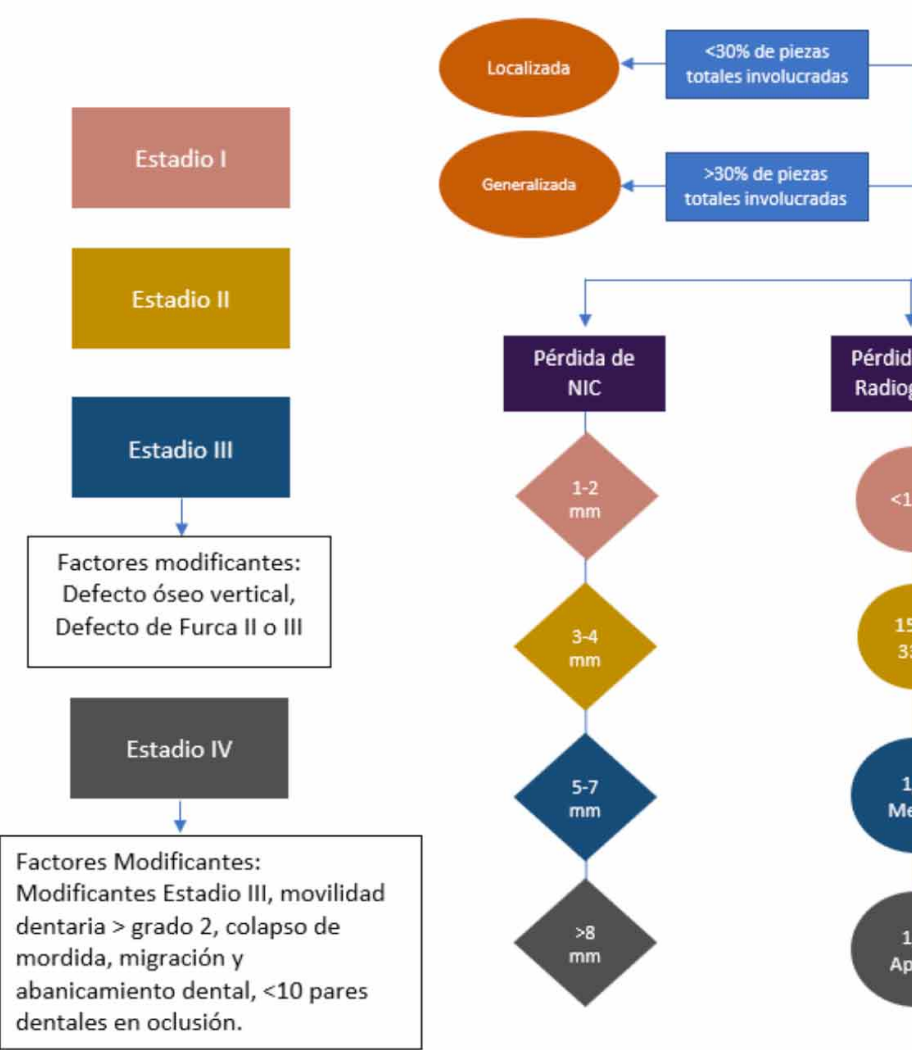

Extensión

Paciente Periodonta

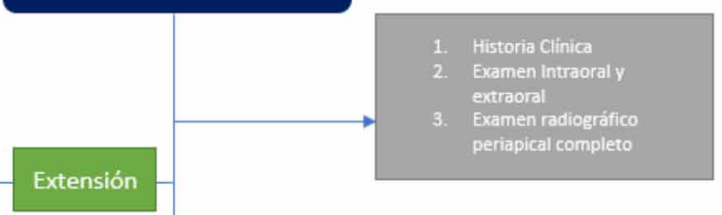

Estadio

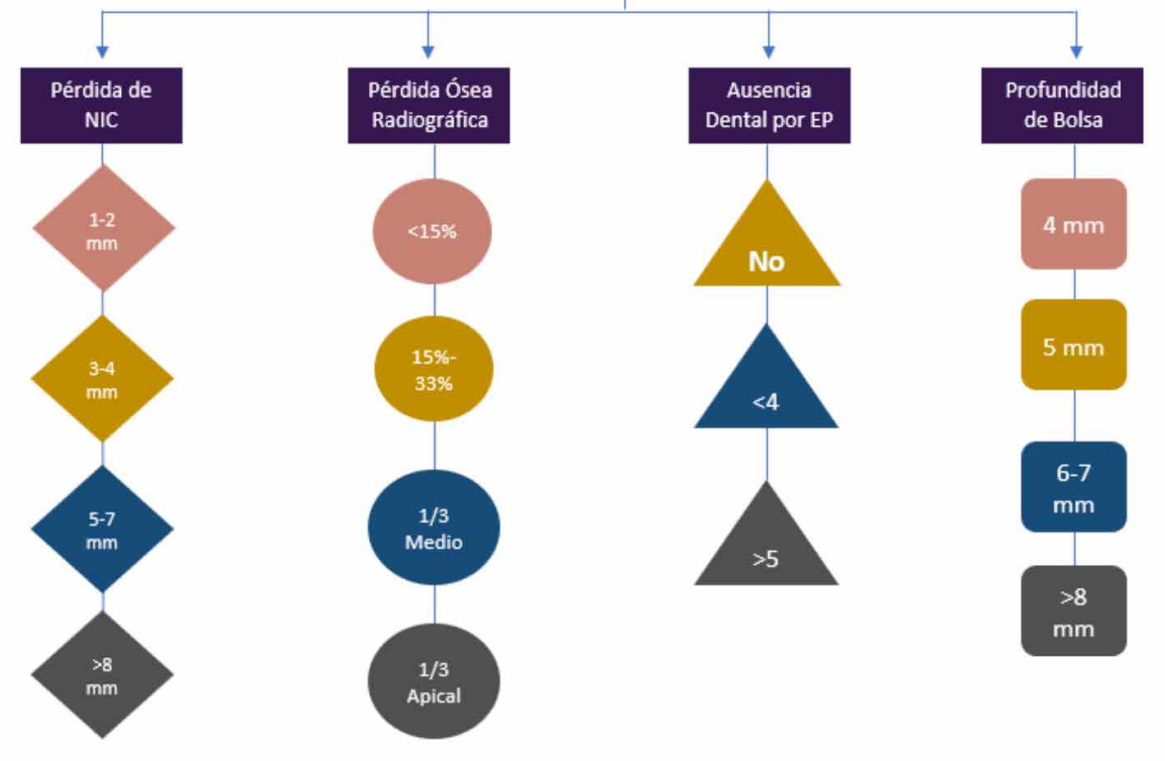

Fig. 1. Diagrama de diagnóstico, adaptado de Tonetti et al. (2018) y Herrera et al. (2018). NIC: nivel de inserción clínico; EP: Enfermedad Periodontal.

tamos dificultades al momento de realizar el diagnostico periodontal, no fue sino después de la calibración y estandarización de la clasificación, que logramos obtener buenos resultados, por lo que la realización de este trabajo pretende destacar los puntos más importantes a considerar de modo que sea un apoyo para el clínico al momento de establecer el diagnostico.

Criterios diagnósticos. La salud gingival se define como la ausencia de sangrado al sondeo, enrojecimiento, edema, perdida de inserción y perdida ósea, niveles óseos reducidos y perdida de inserción clínica (Chapple et al., 2018).

Gingivitis. La gingivitis se puede dividir en 2 grupos principalmente: inducida y no inducida por placa, esta última se refiere a aquellas condiciones y trastornos que no son iniciados por el acúmulo de placa dentobacteriana, pueden ser manifestaciones de ciertas enfermedades sistémicas (Holmstrup et al., 2018). La gingivitis inducida por placa dentobacteriana se identifica como una inflamación debido al acúmulo de placa confinada solo al tejido gingival sin presencia de pérdida de inserción y ósea, es reversible después del tratamiento óptimo, que es la eliminación de placa y cálculo. Se define como un caso de gingivitis cuando se encuentran los siguientes signos; sangrado al sondeo $(>10 \%$ de sitios sangrantes del total de piezas dentales presentes), inflamación gingival donde comúnmente se pierde la anatomía afilada de las papilas interdentales, enrojecimiento, profundidad de sondeo $<3 \mathrm{~mm}$ y el paciente puede referir síntomas como incomodidad, sangrado al cepillado, halitosis y sabor metálico (Herrera et al.). El porcentaje de sangrado al sondeo se obtiene mediante el uso de una sonda periodontal estandarizada, introduciéndola en el surco gingival en 6 lugares de cada órgano dentario (mesial, medial y distal por vestibular y lingual/palatino) con una fuerza de $0.25 \mathrm{~N}$, se obtiene el porcentaje de sangrado, se define como gingivitis localizada si se encuentran $10-30 \%$ de sitios sangrantes y generalizada si es > al $30 \%$ (Chapple et al.). 
Periodontitis. El diagnóstico periodontal debe basarse en una historia clínica médica y dental completa, exámenes intra y extraorales, así como serie radiográfica periapical, todo esto para identificar la enfermedad periodontal y las causas de reabsorción ósea independientes de la periodontitis (alargamientos de corona, lesiones endo-periodontales, ortodoncia, etc.) El diagnóstico diferencial además contempla los signos y síntomas específicos que indican enfermedades periodontales necrosantes y la presencia o ausencia de comorbilidades sistémicas que alteran la respuesta inmune del paciente (Tonetti et al., 2018; Dietrich et al.).

Las principales características de la periodontitis son; pérdida de los tejidos de soporte debido a la inflamación, inserción mayor de $3 \mathrm{~mm}$ y disminución de las crestas óseas evaluada por medio de radiografías, presencia de bolsas periodontales $(>3 \mathrm{~mm}$ ) y sangrado gingival. Una vez que se confirma un caso de periodontitis, se procede a clasificarlo en extensión, estadio y grado (Papapanou et al.).

Estadio y extensión. Se refiere a la severidad de la enfermedad y la complejidad del tratamiento, la primera se basa en la información de la pérdida de inserción, destrucción ósea visible radiográficamente y ausencias dentales asociado a esto; la complejidad del tratamiento incluye la profundidad de las bolsas periodontales, el tipo de defecto óseo (horizontal y vertical), involucro de furca, trauma oclusal, colapso de mordida, abanicamiento dental, entre otros. La extensión se determina evaluando la proporción de piezas con defectos óseos y el nivel de inserción clínica en relación con sitios sanos, si el total de órganos dentarios que presenta estos criterios es mayor al $30 \%$ se denomina periodontitis generalizada, si por el contrario estas características se observan en menos del $30 \%$, es considerada localizada. Por ejemplo, si el paciente cuenta con 28 dientes y se observa pérdida ósea radiográfica y de inserción al momento de realizar el sondeo ( 6 sitios por pieza, en total 168) en 84 sitios, el resultado es 50 $\%$ y la periodontitis es generalizada (Herrera et al.).

Estadios I y II. Estos estadios corresponden al límite entre gingivitis y periodontitis, su asignación depende de la cantidad de pérdida ósea y de inserción clínica, en el estadio I la ausencia de hueso debe ser menor o igual al $15 \%$ de la longitud de la raíz de la pieza dental con mayor defecto (esta se identifica en el examen radiográfico) y la desinserción clínica no debe ser mayor a 1-2 mm, para el estadio II estos criterios son $15-33 \%$ y 3-4 mm respectivamente.
Estadios III y IV. En el estadio III la reabsorción ósea llega al tercio medio o más allá y la pérdida de inserción es igual o mayor a $5 \mathrm{~mm}$, debe de haber ausencias dentales (hasta 4 piezas por razones periodontales) y presencia de más de 10 pares de dientes en oclusión.

En el tipo IV se puede apreciar un faltante aun mayor (más de 4 piezas dentales), y es evidente la reducción del número de dientes en oclusión (menor de 10), además del colapso de la mordida, migraciones dentales y abanicamiento (Tonetti \& Sanz, 2019), aquí estamos hablando de un daño irreparable al tejido periodontal, aumentando la complejidad del tratamiento debido a bolsas periodontales que se extienden a la mitad o más allá de la superficie radicular, defectos intraóseos e involucro de furca, haciendo que se reduzcan las opciones de rehabilitación (Tonetti et al.).

Grado del padecimiento. La periodontitis puede tener un ritmo de progreso distinto en cada paciente y la respuesta del tratamiento puede ser menos predecible o ser afectado por condiciones sistémicas; el objetivo de esta clasificación es utilizar la información disponible para determinar progresos rápidos de la enfermedad en ciertos individuos y modificar tratamientos de acuerdo con esta información. El grado nos brinda conocimiento de las características biológicas de la enfermedad debido a que tiene un origen multifactorial que puede determinar la respuesta del huésped (genéticos, ambientales y comportamiento). Se utilizan como datos el porcentaje de pérdida ósea, la edad del paciente y factores de riesgo como tabaquismo y diabetes, entre otros (Tonetti et al.; Tonetti \& Sanz; Dietrich et al.).

Evidencia Directa (Radiografías Previas Disponibles). Si se cuenta con el registro radiográfico de 5 años de seguimiento, se utiliza la pieza dental con mayor reabsorción ósea para comparar las radiografías actuales; si no existen cambios se considera grado A, si la diferencia entre ellas es menor a $2 \mathrm{~mm}$ se asigna como grado $B$, y cuando es mayor a $2 \mathrm{~mm}$ se categoriza como grado $\mathrm{C}$. Con respecto a la extensión de la periodontitis, se elige el área con mayor defecto y a partir de esta se compara o se evalúa dependiendo de la información con la se cuenta (evidencia directa o indirecta). El grado A y B pueden agravarse y convertirse en $\mathrm{C}$ si el paciente cuenta con factores modificadores (ser fumador de 10 o más cigarrillos al día y presentar niveles de hemoglobina glicosilada (HbA1c) mayor a 7. 


\section{Evidencia Indirecta (Sin Registros Radiográficos} Disponibles). En este caso, al no existir registros para comparar, los criterios se tomarán del área con mayor defecto óseo con respecto a la medida de la raíz de la pieza involucrada, de acuerdo con Tonetti et al., se realiza una división matemática entre el porcentaje de pérdida de hueso en relación con la longitud radicular y la edad del paciente (PO/E). Si el resultado es $<0,25$ es grado $A$, grado $B$ si es entre 0,25 y 1,0 y grado $C$ si es $>1$, si el porcentaje de un paciente en el área designada es $60 \%$ y la edad del paciente es de 45 años, la división sería 60/45=1,33; el grado correspondiente sería el C. Además, se consideran la presencia de depósitos de placa dentobacteriana y la relación de estos con el patrón de destrucción, el grado se puede modificar de igual manera por factores de riesgo previamente descritos en la evidencia directa, como se muestra en las Figuras 1 y 2 (Herrera et al.).

\section{CONCLUSIÓN}

La nueva clasificación tiene como objetivo agrupar las enfermedades gingivales y periodontales de una manera más sencilla, como todo cambio en un inicio puede resultar confuso, para nosotros no fue la excepción, ya que experimentamos variaciones diagnósticas al momento de realizar nuestras actividades profesionales y académicas, por lo que decidimos trabajar al respecto, realizamos la estandarización de la técnica y nos calibramos tanto profesores como alumnos hasta lograr realizar el diagnóstico de manera adecuada, en este trabajo presentamos los puntos más importantes que consideramos para lograr dicho propósito y los cuales pretendemos sean un apoyo para el odontólogo tanto de practica general como para el especialista al momento de establecer el diagnostico.

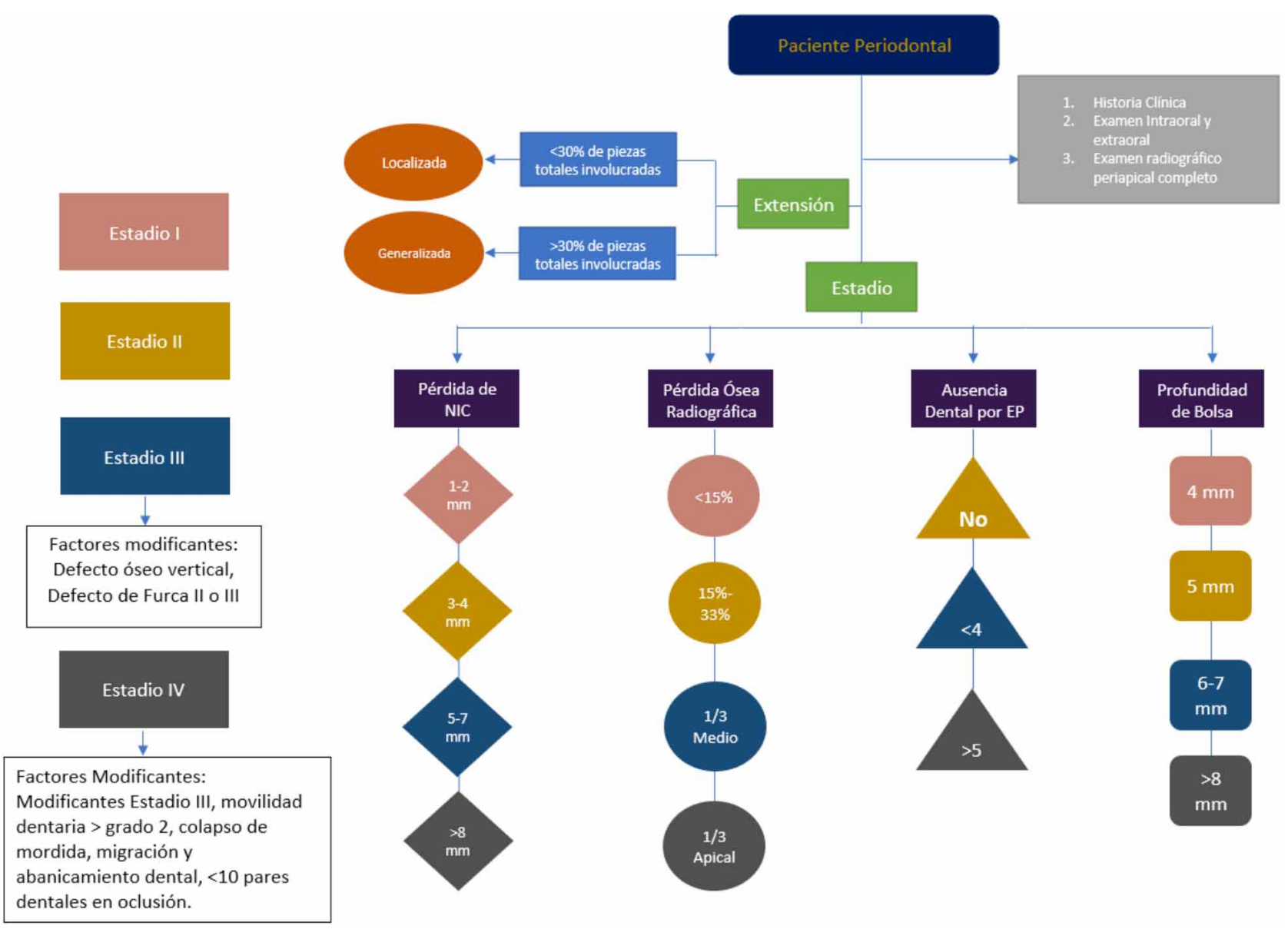

Fig. 2. Compendio de criterios a evaluar para realizar el correcto diagnóstico de enfermedad periodontal, adaptado de Tonetti et al. (2018) y Herrera et al. (2018). NIC: nivel de inserción clínico; EP: Enfermedad Periodontal. 
CÁRDENAS, V. P.; GUZMÁN, G. D. A.; VALERA, G. E. ; CUEVAS, G. J. C.; ZAMBRANO, G. G. \& GARCÍA, C. A. G. Main diagnostic criteria of the new classification of conditions and periodontal diseases. Int. J. Odontostomat., 15(1):175$180,2021$.

ABSTRACT: Having a classification of periodontal conditions and diseases is a very useful tool for clinicians by providing a systematic guide to different manifestations of periodontal issues. The 2017 classification of periodontal diseases and conditions has as one of the main characteristics the division of the Periodontitis group classified by stage, which assigns the severity and complexity of the treatment, and by grade, which refers to the rhythm of progression and the risk of future relapse; periodontitis as a manifestation of systemic conditions and necrotizing periodontal diseases. As well as the featuring of peri-implant health and diseases. A more didactic route of diagnostic will be given in this publication and the main differences between the last two classifications will be explained, while we share the experience of implementing this new classification in our institute.

KEY WORDS: periodontitis, chronic periodontitis, periapical.

\section{REFERENCIAS BIBLIOGRÁFICAS}

Armitage, G. C. Development of a classification system for periodontal diseases and conditions. Ann. Periodontol., 4(1):16, 1999.

Caton, J. G.; Armitage, G.; Berglundh, T.; Chapple, I. L. C.; Jepsen, S.; Kornman, K. S.; Mealey, B. L.; Papapanou, P. N.; Sanz, M. \& Tonetti, M. S. A new classification scheme for periodontal and peri-implant diseases and conditions - Introduction and key changes from the 1999 classification. J. Clin. Periodontol., 45 Suppl. 20:S1-8, 2018.

Chapple, I. L. C.; Mealey, B. L.; Van Dyke, T. E.; Bartold, P. M:; Dommisch, H.; Eickholz, P.; Geisinger, M. L.; Genco, R. J.; Glogauer, M.; Goldstein, M.; et al. Periodontal health and gingival diseases and conditions on an intact and a reduced periodontium: Consensus report of workgroup 1 of the 2017 World Workshop on the Classification of Periodontal and PeriImplant Diseases and Conditions. J. Clin. Periodontol., 45 Suppl. 20:S68-77, 2018.

Dietrich, T.; Ower, P.; Tank, M.; West, N. X.; Walter, C.; Needleman, I.; Hughes, F. J.; Wadia, R.; Milward, M. R.; Hodge, P. J.; et al. Periodontal diagnosis in the context of the 2017 classification system of periodontal diseases and conditions - implementation in clinical practice. Br. Dent. J., 226(1):16-22, 2019.

Graetz, C.; Mann, L.; Krois, J.; Sälzer, S.; Kahl M.; Springer, C. \& Schwendicke, F. Comparison of periodontitis patients' classification in the 2018 versus 1999 classification. J. Clin. Periodontol., 46(9):908-17, 2019.

Herrera, D.; Figuero, E.; Shapira, L.; Jin, L. \& Sanz, M. La Nueva clasificación de enfermedades periodontales y periimplantares. Rev. Cient. Soc. Esp. Periodoncia, 11, 2018.

Holmstrup, P.; Plemons, J. \& Meyle, J. Non-plaque-induced gingival diseases. J. Periodontol., 89 Suppl. 1:S28-45, 2018.
Papapanou, P. N.; Sanz, M.; Buduneli, N.; Dietrich, T.; Feres, M.; Fine, D. H.; Flemmig, T. F.; Garcia, R.; Giannobile, W. V.; Graziani, F.; et al. Periodontitis: Consensus report of workgroup 2 of the 2017 World Workshop on the Classification of Periodontal and Peri-Implant Diseases and Conditions. J. Periodontol., 89 Suppl. 1:S173-82, 2018.

Tonetti, M. S. \& Sanz, M. Implementation of the new classification of periodontal diseases: Decision-making algorithms for clinical practice and education. J. Clin. Periodontol., 46(4):398-405, 2019.

Tonetti, M. S.; Greenwell, H. \& Kornman, K. S. Staging and grading of periodontitis: Framework and proposal of a new classification and case definition. J. Periodontol., 89 Suppl. 1:S159-72, 2018.

Dirección para correspondencia:

Mtra. Alma Graciela García Calderón

Av. Benjamín Franklin no. 4650

zona PRONAF CP 32315

Ciudad Juárez Chihuahua

MÉXICO

E-mail: alma.garcia@uacj.mx 